\title{
Review: capillary refill time, abnormal skin turgor, and abnormal respiratory pattern are useful signs for detecting dehydration in children
}

Steiner MJ, DeWalt DA, Byerley JS. Is this child dehydrated? JAMA 2004;291:2746-54.

What is the accuracy of signs, symptoms, and laboratory tests for detecting dehydration in children?

\section{METHODS}

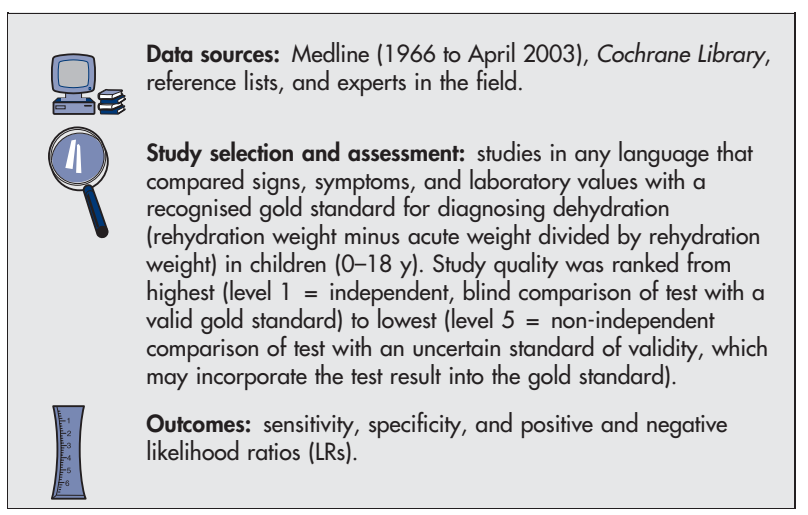

\section{MAIN RESULTS}

13 studies $(\mathrm{n}=1246)$ met the selection criteria (4 intermediate quality and 9 low quality). Signs that were clinically useful for detecting 5\% dehydration were prolonged capillary refill time, abnormal skin turgor, and abnormal respiratory pattern (table) Dry mucous membranes, sunken eyes, and poor overall appearance were moderately useful in detecting $5 \%$ dehydration (table). 3 studies evaluated combinations of signs. In l study $(\mathrm{n}=100)$, the combination of abnormal skin turgor, sunken eyes, dry mucous membranes, and a sunken fontanelle increased the likelihood of $10 \%$ dehydration being present (LR 3.7, 95\% CI 1.6 to 8.1). Another study $(\mathrm{n}=97)$ showed that classification of "severe" on an assessment scale detected $\geqslant 5 \%$ dehydration in children who required intravenous fluids (LR 3.4, CI 1.5 to 7.7 ). In the third study $(\mathrm{n}=225)$, the combination of $\geqslant 3$ of 10 signs was useful for detecting $5 \%$ dehydration (sensitivity $87 \%$, specificity $82 \%$ ). In a pooled analysis of 3 studies ( $\mathrm{n}=398$ ), history taking (eg, parental report of low urine output) was not accurate for detecting the likelihood of 5\% dehydration (LR 1.3, CI 0.9 to 1.9). Hill School of Medicine, Chapel Hill, NC, USA. dewaltd@med.unc.edu Source of funding: Robert Wood Johnson Clinical Scholars Program.

\section{CONCLUSIONS}

Prolonged capillary refill time, abnormal skin turgor, and abnormal respiratory pattern are the most useful signs for detecting dehydration in children. Combinations of signs perform better than individual signs.

\section{A modified version of this abstract appears in Evidence-Based Medicine.}

\section{Commentary}

D ehydration is associated with the leading causes of morbidity and mortality in children throughout the world. ${ }^{1}$ Nurses in various clinical contexts must routinely assess the hydration status of paediatric patients. Identifying the best indicators or combinations of indicators of children's hydration status will enable practitioners to optimise patient management and minimise unnecessary procedures.

The review by Steiner et al gives credence to the use of some clinical signs when assessing children's hydration status. Although the authors did a thorough literature search, most of the 13 included studies had low methodological quality (unblinded non-independent comparison of tests with a valid gold standard in a selected sample of patients). The findings are current best evidence but should still be viewed with some caution.

The findings are relevant to paediatric practitioners in both outpatient and inpatient settings. The review indicates that current assessment of hydration is extremely variable and may not be well founded. For example, practitioners often turn to parents for information on a child's illness, but parental report was found to be a poor indicator of children's hydration status.

The review by Steiner et al showed that the clinical examination is the most important method of assessing hydration in children. Rating scales incorporating combinations of signs have been developed to expedite diagnosis ${ }^{2}$ but may not include the combinations this review has identified as being the most predictive of dehydration. Future efforts at developing scales or scoring systems should evaluate the accuracy of signs identified in this review using the best evaluative methods for diagnostic testing.

Tina Popov, RN, MN, ACNP Hospital for Sick Children Toronto, Ontario, Canada

1 King CK, Glass R, Bresee JS, et al. Managing acute gastroenteritis among children: oral rehydration, maintenance, and nutritional therapy. MMWR Recomm Rep 2003;52:1-16.

2 Friedman JN, Goldman RD, Srivastava R, et al. Development of a clinical dehydration scale for use in children between 1 and 36 months of age. Pediatr 2004;145:201-7.

Test characteristics of examination signs to detect $5 \%$ dehydration in children (age range 2 wks to $15 \mathrm{y}$ )

\begin{tabular}{llllll}
\hline Examination signs & Number of studies $(\mathbf{n})$ & Sensitivity (95\% CI) & Specificity (CI) & +LR & - LR \\
\hline Prolonged capillary refill & $4(478)$ & $60 \%(29$ to 91$)$ & $85 \%(72$ to 98$)$ & 4.0 & 0.47 \\
Abnormal skin furgor & $5(602)$ & $58 \%(40$ to 75$)$ & $76 \%(59$ to 93$)$ & 2.42 & 0.55 \\
Abnormal respiratory pattern & $4(581)$ & $43 \%(31$ to 55$)$ & $79 \%(72$ to 86$)$ & 2.05 & 0.72 \\
Dry mucous membranes & $4(533)$ & $86 \%(80$ to 92$)$ & $44 \%(13$ to 74$)$ & 1.54 & 0.32 \\
Sunken eyes & $4(533)$ & $75 \%(62$ to 88$)$ & $52 \%(22$ to 81$)$ & 1.56 & 0.48 \\
Poor overall appearance & $3(398)$ & $80 \%$ (57 to 104) & $45 \%$ (-10 to 102) & 1.45 & 0.44 \\
\hline
\end{tabular}

* $L R=$ likelihood ratio; other abbreviations defined in glossary. Sensitivity, specificity, $+L R$, and $-L R$ were calculated from data in article using a random effects model. 